\title{
Analysis of the Antimicrobial Efficacy of Bamboo/Cotton Knitted Fabric Finished with the Extracts of the Syzygium aromaticum Buds
}

\section{Ramya $\mathrm{K}^{1 *}$ and Maheshwari $\mathrm{V}^{2}$}

${ }^{1,2}$ Department of Costume Design \& Fashion, PSG College of Arts \& Science, Coimbatore, TN, India

\begin{abstract}
The present study aims at the development of eco-friendly antimicrobial finished Bamboo/Cotton fabrics from the herbal extracts. The herbal extracts of Syzygium aromaticum (clove) buds have been applied on to the fabrics by two methods: Direct application method and Microencapsulation Method. The antimicrobial efficacy and the wash durability are assessed using AATCC 147, AATCC 30 and EN ISO 20645 standards. Both the treatments showed good antibacterial property and the microencapsulation method showed good antifungal property too. The Direct application method showed wash durability only till 10 washes, whereas the microencapsulation method showed very good wash durability till 30 wash cycles.
\end{abstract}

Keywords: Antimicrobial finishing; Herbs; Microencapsulation; Wash durability

\section{Introduction}

Recently there has been upsurge interest in apparel technology all over the world for much demanding functionality of the products like wrinkle resistance, water repellence, fade resistance and resistance to the microbial invasion. Among these, development of antimicrobial textile finish is highly indispensable and relevant since garments are in direct contact with the human body [1]. The textile finishes with added values particularly for medical cloths are greatly appreciated and the rapid growth in the field of medical textiles and their end uses has generated many opportunities for the application of antimicrobial finishes. The consumers are aware of hygienic life style and there is a necessity of textile products with antimicrobial properties. Several antimicrobial agent's viz., triclosan, quaternary ammonium compounds and recently nano silver are available for textile finishing. However due to their cost and synthetic nature, eco-friendly plant extracts are gaining significant momentum [2].

Recognising the importance of plant materials as antimicrobial agents, research has been initiated in the areas of producing bioactive textiles for the protection of wearer from common microbes causing cross infections. Natural antimicrobial agents are non-toxic and nonallergenic and do not cause the problems of microbial resistance [3]. There is a growing interest in plants with antimicrobial activity. Scientists are increasingly becoming involved in the screening of such plants with the aim of establishing their potential antimicrobial effects and identifying the compounds responsible for the antimicrobial properties [4].

Therefore it is an indispensible need for more investigation in the development of eco-friendly antibacterial agents extracted from plants for textile applications. Although certain natural antibacterial agents are available at present, only few studies have been explored for their antibacterial activity on textile materials and also require progressive and consolidated data on antimicrobial finished product of textiles particularly in the preparation of medical cloths [5].

As garments are subjected to washing, the wash durability of finishes is a major issue. Even though many of the herbal extracts have shown good antimicrobial property after applying on textile fabrics, their wash durability is poor. Hence in this study microencapsulation technique was used to fix the herbal extracts on the fabrics. Microencapsulation is one of the novel methods of getting functional finishes on textiles. It is a process by which very tiny droplets of liquid or particles of solid are covered with a continuous film of polymeric material. Microencapsulation is more advantageous to conventional processes in terms of economy, energy saving, eco friendliness and controlled release of substance. The agents reside in colloidal suspension within the amorphous zone of the binder so that a reservoir of agent is preventing in solid solution within the polymer matrix. Such treated fabrics were reported to be durable to a few number of wash cycles [6].

The present study aims at developing an eco-friendly and natural antimicrobial finish on textile fabrics using the extracts of Syzygium aromaticum buds (clove buds). Syzygium aromaticum (cloves buds) are the aromatic dried flower buds of a tree in the family Myrtaceae and are used worldwide as important spice of kitchen. The extracts were applied to the bamboo/cotton knitted fabric by direct application method and microencapsulation method. An extensive study was conducted to assess the antimicrobial effectiveness and their wash durability by employing standard test methods.

\section{Materials and Methods}

For the purpose of the study, 50:50 Bamboo-Cotton yarns of 40's count were knitted with Single Jersey structure was selected. The medicinal herbs Syzygium aromaticum (cloves buds) was chosen and collected from the Nilgiris district for the study.

\section{Herbal extraction}

The collected buds were cleaned and grind into a fine powder. For extraction, $3 \mathrm{~g}$ of dry powder was taken and mixed into $50 \mathrm{ml}$ of $80 \%$ Methanol. The container was closed and kept for overnight. After overnight incubation, the extract was filtered through filter paper and evaporated to concentrate the extract.

*Corresponding author: Ramya K, Department of Costume Design \& Fashion, PSG College of Arts \& Science, Coimbatore, TN, India, E-mail: ramyakathiresan@yahoo.com

Received July 10, 2013; Accepted August 21, 2013; Published August 26, 2013

Citation: Ramya K, Maheshwari V, (2013) Analysis of the Antimicrobial Efficacy of Bamboo/Cotton Knitted Fabric Finished with the Extracts of the Syzygium aromaticum Buds. J Textile Sci Eng 3: 134. doi:10.4172/2165-8064.1000134

Copyright: @ 2013 Ramya K, et al. This is an open-access article distributed under the terms of the Creative Commons Attribution License, which permits unrestricted use, distribution, and reproduction in any medium, provided the original author and source are credited. 
Citation: Ramya K, Maheshwari V, (2013) Analysis of the Antimicrobial Efficacy of Bamboo/Cotton Knitted Fabric Finished with the Extracts of the Syzygium aromaticum Buds. J Textile Sci Eng 3: 134. doi:10.4172/2165-8064.1000134

Page 2 of 5

\section{Finishing of the fabrics using extracts}

Direct application method: The prepared methanol extract were directly applied on the Bamboo/Cotton fabric using pad-dry-cure method.

Microencapsulation method: Micro capsules containing herbal extracts were prepared employing Sodium alginate. 3\% Sodium alginate solution was prepared using distilled water. Equal proportion of sodium alginate and extracts was prepared separately. This was sprayed into calcium chloride solution by means of a sprayer. The droplets were retained in calcium chloride for 15 minutes. The microcapsules were obtained by decantation and repeated washing with isopropyl alcohol followed by drying at $45 \mathrm{UC}$ for $12 \mathrm{hrs}$. The microcapsules were then used for finishing on the Bamboo/Cotton fabrics by exhaustion method using $8 \%$ citric acid as binder at $50^{\circ} \mathrm{C}$ for 30 minutes.

The finished Bamboo/Cotton fabrics were assessed as per the AATCC 147, EN ISO 20645 and AATCC 30 standards for the antimicrobial activity against the standard microorganisms; Gram Positive-Staphylococcus aureus and Gram Negative-Escherichia coli.

\section{Assessment of the antibacterial efficacy using AATCC 147 standards}

The Bamboo/Cotton fabric samples were cut in rectangular shape with $25 \times 50 \mathrm{~mm}$ for analysis. Sterile bacteriostasis agar was dispensed in to Petri dishes. Broth cultures ( 24 hours) of the test organisms were used as inoculums. Using sterile inoculation loop, the test organisms (Escherichia coli \& Staphylococcus aureus) were streaked, 5 lines with 4 $\mathrm{mm}$ width over the surface of the agar plate. Pre-sterilized samples were placed over the culture inoculated agar surface by using sterile forceps. After placing the samples, all the plates were incubated at $37^{\circ} \mathrm{C}$ for 18 to 24 hours. After incubation, the plates were examined for the zone of bacterial inhibition around the fabric sample. The size of the clear zone was used to evaluate the inhibitory effect of the sample.

\section{Assessment of the antifungal efficacy using AATCC 30 standards}

An inoculum of $1.0 \mathrm{ml}$ was evenly distributed over the surface of the agar. The fabric discs were pre wetted (not rubbed or squeezed) in water containing $0.05 \%$ of a non-ionic wetting agent (triton X-100) and placed on the agar surface. The inoculum of $0.2 \mathrm{ml}$ was distributed evenly over each disc by means of a sterile pipette. All the specimens were incubated at a temperature of $28^{\circ} \mathrm{C}$ for seven days.

At the end of the incubation period, the percentage of the surface area of the disc covered with the growth of the fungus was reported by observing visually and using a microscope (40X) and interpreted as follows:

1. No growth (If present, the size of the growth free zone in $\mathrm{mm}$ was reported)

2. Microscopic growth (visible only under the microscope)

3. Macroscopic growth (visible to the naked eye).

\section{Assessment of the wash durability using EN ISO 20645 standards}

The antibacterial activity of the finished Bamboo/Cotton fabric was tested according to EN ISO 20645 against Staphylococcus sp. and Escherichia coli. Nutrient agar plates were prepared by pouring $15 \mathrm{ml}$ of media into sterile Petri dishes. The agar plates were allowed to solidify for 5 minutes and $0.1 \%$ inoculum was swabbed uniformly and allowed to dry for 5 minutes. The finished fabric with the diameter of $2.0 \pm 0.1$ $\mathrm{cm}$ was placed on the surface of medium and the plates were kept for incubation at $37^{\circ} \mathrm{C}$ for 24 hours. At the end of incubation, the zone of inhibition formed around the fabric was measured in millimeters and recorded.

\section{Results and Discussions}

\section{Assessment of antibacterial efficacy using AATCC 147 standards}

Antibacterial activities of the Syzygium aromaticum extract treated

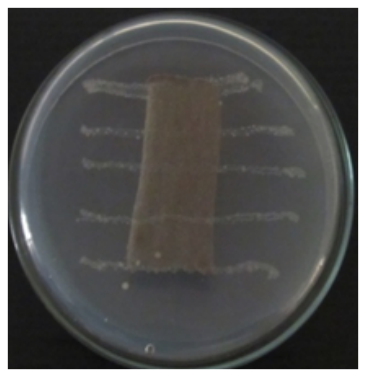

Figure 1a: Untreated (control) against S. aureus.

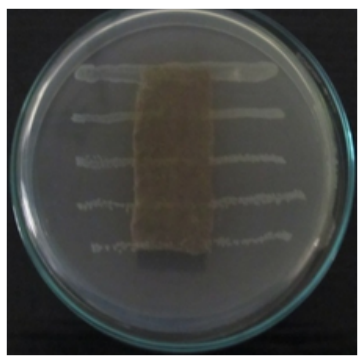

Figure 1b: Untreated (control) against E. coli.

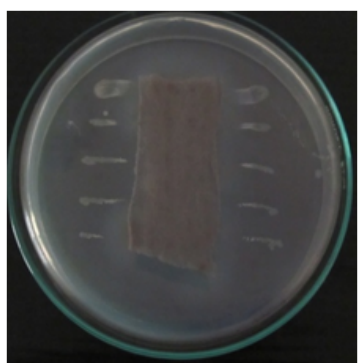

Figure 1c: Treated sample by direct application against $S$. aureus.

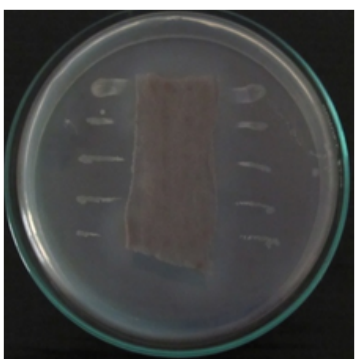

Figure 1d: Treated sample by direct application against $E$. coli 
Citation: Ramya K, Maheshwari V, (2013) Analysis of the Antimicrobial Efficacy of Bamboo/Cotton Knitted Fabric Finished with the Extracts of the Syzygium aromaticum Buds. J Textile Sci Eng 3: 134. doi:10.4172/2165-8064.1000134

Page 3 of 5

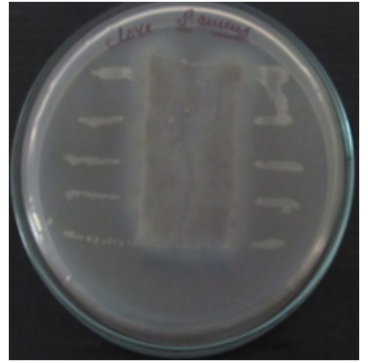

Figure 1e: Treated sample by microencapsulation against $S$. aureus.

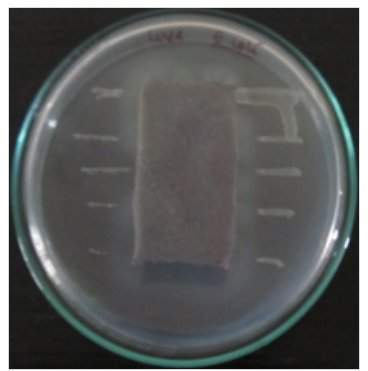

Figure 1f: Treated sample by microencapsulation against $E$. coli.

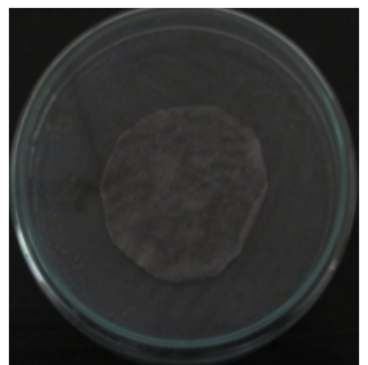

Figure 2a: Untreated fabric against Aspergillus niger.

Bamboo/Cotton fabrics using AATCC 147 method are given in Table 1 and Figures 1a-f. The Parallel streak method results clearly shows that the untreated (control) sample showed growth under the test specimen and the treated fabrics have very good antibacterial efficacy against both the Gram positive and Gram negative microorganisms. The treated samples do not have growth under the specimens and the zone of inhibition against the Gram positive ranges from 31 to $36 \mathrm{~mm}$ and against Gram Negative ranges from 29 to $35 \mathrm{~mm}$.

\section{Assessment of antifungal efficacy and wash durability using AATCC 30 standards by direct application and microencapsulation method}

The antifungal properties of the treated Bamboo/Cotton fabrics and their wash durability for few wash cycles are shown in the Table 2 and Figures 2a-i. It is clearly noted that samples treated with Syzygium aromaticum extract by direct application method do not possess antifungal properties, but the samples treated by microencapsulation method shows very good antifungal property and withstands 20 wash cycles.

\section{Assessment of Wash Durability by EN ISO 20645}

Table 3 and Figures 3a-c shows that Syzygium aromaticum extract when directly treated on the Bamboo/Cotton fabrics showed good antibacterial efficiency against both the microorganisms but showed poor wash durability. This indicates that the direct treatment on the fabric surface, get easily removed by washing.

The Table 3 and Figures 3d-e also indicates that the samples treated by microencapsulation method showed excellent antibacterial property against both the microorganisms and also showed very good wash durability up to 30 washes. The antibacterial effect of the microencapsulated fabric is much affected by repeated washes when compared to the direct application method.

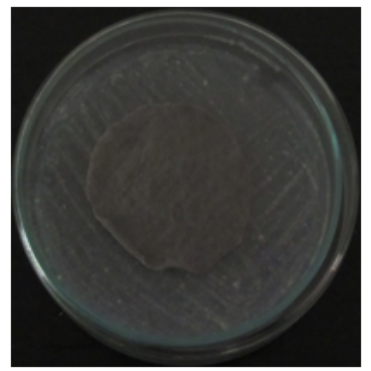

Figure 2b: Fabric treated Syzygium aromaticum extract ( 0 wash) by direct application method.

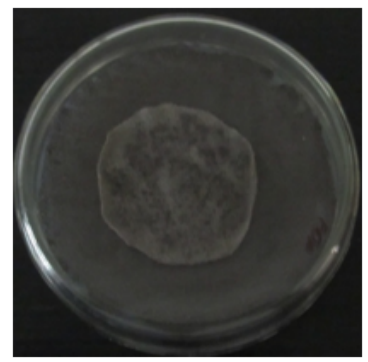

Figure 2c: Fabric treated by direct application \& washed (10 washes).

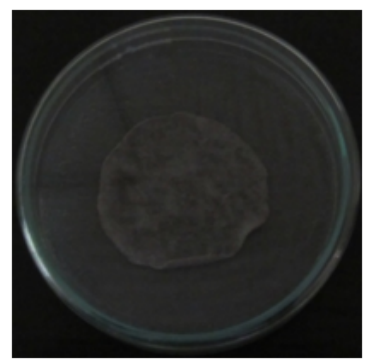

Figure 2d: Fabric treated by direct application \& washed (20 washes).

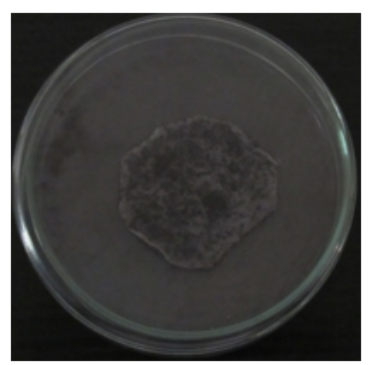

Figure 2e: Fabric treated by direct application \& washed (30 washes) 
Citation: Ramya K, Maheshwari V, (2013) Analysis of the Antimicrobial Efficacy of Bamboo/Cotton Knitted Fabric Finished with the Extracts of the Syzygium aromaticum Buds. J Textile Sci Eng 3: 134. doi:10.4172/2165-8064.1000134

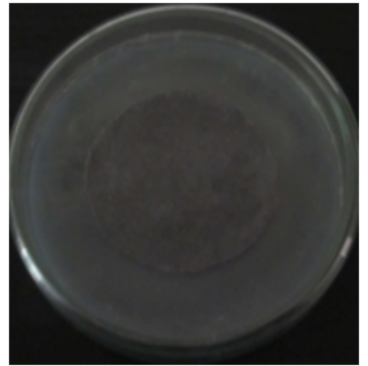

Figure 2f: Fabric treated with Syzygium aromaticum extract by microencapsulation (0 wash)

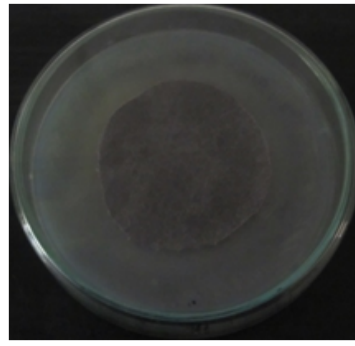

Figure 2g: Fabric treated by microencapsulation \& washed (10 washes).

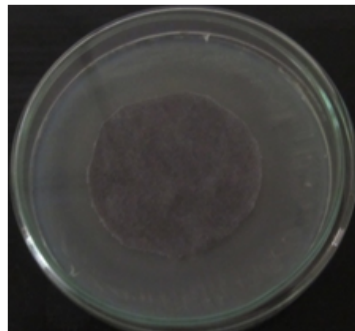

Figure $\mathbf{2 h}$ : Fabric treated by microencapsulation \& washed (20 washes).

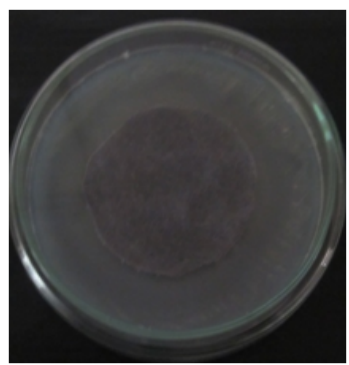

Figure 2i: Fabric treated by microencapsulation \& washed (30 washes).

\section{Conclusion}

The fabrics samples treated with Syzygium aromaticum extracts showed considerable zone of inhibition against Gram positiveStaphylococcus aureus and Gram negative-Escherichia coli bacteria. There was no bacterial growth seen on the treated fabrics (zone of inhibition ranges from $29 \mathrm{~mm}-36 \mathrm{~mm}$ ). The extracts are directly applied on to the fabric by pad-dry-cure method, but the only disadvantage is that, the wash durability of the directly treated fabrics is poor. But the fabrics treated by microencapsulation method, showed good antimicrobial (antibacterial and antifungal) property and also good

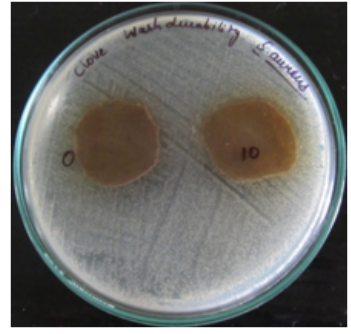

Figure 3a: Fabric Treated by direct application and washed fabrics 0 and 10 wash against $S$. aureus.

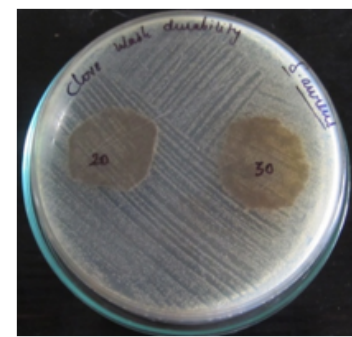

Figure 3b: Fabric treated by direct application and washed fabrics 20 and 30 washes against $S$. aureus.

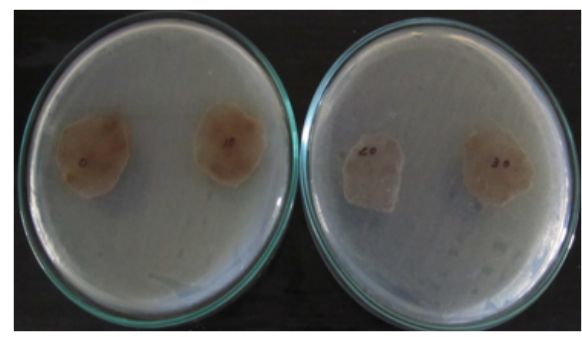

Figure 3c: Fabric treated by direct application and washed fabrics 20 and 30 washes against E.coli.

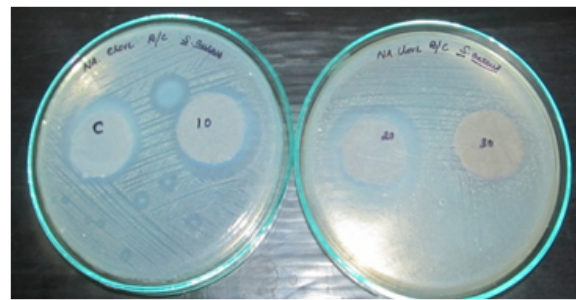

Figure 3d: Fabric treated microencapsulation and washed fabrics 0,10 20 and 30 washes against $S$. aureus.

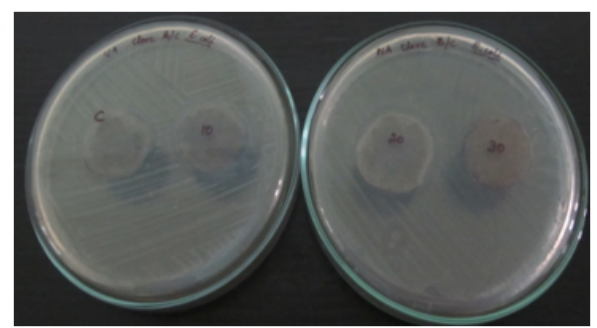

Figure 3e: Fabric treated microencapsulation and washed fabrics 0,10 20 and 30 washes against E.coli. 
Citation: Ramya K, Maheshwari V, (2013) Analysis of the Antimicrobial Efficacy of Bamboo/Cotton Knitted Fabric Finished with the Extracts of the Syzygium aromaticum Buds. J Textile Sci Eng 3: 134. doi:10.4172/2165-8064.1000134

Page 5 of 5

\begin{tabular}{|l|l|c|c|c|c|}
\hline Herb & Fabric samples & $\begin{array}{c}\text { Microbial Growth under } \\
\text { fabric }\end{array}$ & \multicolumn{2}{|c|}{$\begin{array}{c}\text { Direct Application method } \\
\text { Zone of Bacteriostasis (mm) }\end{array}$} & $\begin{array}{c}\text { Microencapsulation method } \\
\text { Zone of Bacteriostasis (mm) }\end{array}$ \\
\cline { 2 - 5 } & & Staphylococcus aureus & Escherichia coli & Staphylococcus aureus & Escherichia coli \\
\hline $\begin{array}{l}\text { Syzygium aromaticum } \\
\text { extract finished Bamboo: } \\
\text { Cotton (50:50) }\end{array}$ & Untreated sample & Preated sample & Absent & 0 & 0 \\
\hline
\end{tabular}

Table 1: Assessment of the Syzygium aromaticum buds extract treated fabrics.

\begin{tabular}{|l|c|c|}
\hline Herb & No of Washes & \multicolumn{2}{|c|}{ Zone of Bacteriostasis (mm) Aspergillus niger } \\
\cline { 2 - 3 } & & Direct Application method \\
\hline $\begin{array}{l}\text { Syzygium aromaticum extract finished Bamboo: Cotton } \\
(50: 50)\end{array}$ & 0 wash & 0 \\
\cline { 2 - 3 } & 10 washes & 0 \\
\cline { 2 - 3 } & 20 washes & 0 \\
\hline
\end{tabular}

Table 2: Assessment of antifungal property of the Syzygium aromaticum buds extract treated fabrics.

\begin{tabular}{|c|c|c|c|c|c|}
\hline \multirow[t]{3}{*}{ Herb } & \multirow[t]{3}{*}{ No of Washes } & \multicolumn{4}{|c|}{ Zone of Bacteriostasis (mm) } \\
\hline & & \multicolumn{2}{|c|}{ Direct Application method } & \multicolumn{2}{|c|}{ Microencapsulation method } \\
\hline & & Staphylococcus aureus & Escherichia coli & Staphylococcus aureus & Escherichia coli \\
\hline \multirow{4}{*}{$\begin{array}{l}\text { Syzygium aromaticum extract finished } \\
\text { Bamboo: Cotton (50:50) }\end{array}$} & 0 wash & 25 & 24 & 31 & 29 \\
\hline & 10 washes & 23 & 22 & 32 & 30 \\
\hline & 20 washes & 0 & 0 & 33 & 35 \\
\hline & 30 washes & 0 & 0 & 22 & 22 \\
\hline
\end{tabular}

Table 3: Wash durability of the Syzygium aromaticum treated and washed fabrics against S.aureus and E. coli by direct application method and microencapsulation method.

wash durability. Hence the direct application method is suitable for the fabrics are used for single use applications like Bandage gauze, surgical cloths, baby diapers, sanitary napkins, and for the fabrics that need repeated launderings like garments, bed linens, socks, hospital fabrics, etc., can be microencapsulated.

And also Syzygium aromaticum are easily available all over the world, there is a good opportunity for the implementation and the commercialization of the herbal extracts to impart antimicrobial finish on the textile fabrics. As the extracts are purely from the natural resources, the finished fabric is eco-friendly and satisfies the social and the environmental needs.

\section{References}

1. Deepti G, Ankur L (2007) Antimicrobial activity of cotton fabric treated with
Querus infectoria extract. International Journal of Firer and Textile Research 32: 88-92.

2. Sakthivel S, Kathiresan S, Ezihilanban (2012) Recent developments in antimicrobial finishes. Asian Dyer.

3. Joshi M, Wazed AS, Purwar R (2009) Ecofriendly Antimicrobial Finishing of Textiles using bioactive agents based on natural products. International Journal of Firer and Textile Research 34: 295-304.

4. Mahesh S, Manjunatha AH, Vijaya KG (2011) Studies on Antimicrobial textile finish using certain plant natural products. International Conference on Advances in Biotechnology and Pharmaceutical Sciences, Bangkok, Thailand.

5. Nwinyi OC Chinedu NS, Ajani OO (2008) Evaluation of Antibacterial activity of Pisidium guajava and Gongronema latifolium. Journal of Medicinal Plants Research 2: 18-192

6. Sathianarayanan M P, Bhat NV, Kokate SS, Walnuj VE (2010) Antibacterial finish on Cotton fabric from herbal products. International Journal of Fiber and Textile Research 35: 50-58. 